OPEN ACCESS

Edited by: Daisuke Yabe,

Gifu University, Japan

Reviewed by:

Maria Gazouli,

National and Kapodistrian University of Athens, Greece

Jun Liu,

Fudan University, China

*Correspondence:

Dandan Sun

dan_101912@hotmail.com

Jun Yang

junyang63@sina.com

${ }^{\dagger}$ These authors have contributed equally to this work

Specialty section: This article was submitted to Clinical Diabetes,

a section of the journal

Frontiers in Endocrinology

Received: 26 July 2020 Accepted: 21 December 2020 Published: 08 February 2021

Citation:

Zhu Z, Zhang $Y$, Bai $R$, Yang $R$, Shan Z, Ma C, Yang J and Sun D (2021) Association of Genetic Polymorphisms in MicroRNAs With Type 2 Diabetes Mellitus in a Chinese Population.

Front. Endocrinol. 11:587561. doi: 10.3389/fendo.2020.587561

\section{Association of Genetic Polymorphisms in MicroRNAs With Type 2 Diabetes Mellitus in a Chinese Population}

\author{
Zaihan Zhu ${ }^{1+}$, Yanfen Zhang ${ }^{1 \dagger}$, Ruocen Bai ${ }^{1}$, Ru Yang ${ }^{1}$, Zhongyan Shan ${ }^{2}$, Chunyan $\mathrm{Ma}^{1}$, \\ Jun Yang ${ }^{1 *}$ and Dandan Sun ${ }^{1 *}$ \\ 1 Department of Cardiovascular Ultrasound, The First Affiliated Hospital of China Medical University, Shenyang, China, \\ ${ }^{2}$ Department of Endocrinology and Metabolism, Institute of Endocrinology, Liaoning Provincial Key Laboratory of Endocrine \\ Diseases, The First Affiliated Hospital of China Medical University, Shenyang, China
}

Introduction: MicroRNAs (miRNA) involved in the insulin signaling pathways deeply affect the pathogenesis of T2DM. The aim of this study was to assess the association between single nucleotide polymorphisms (SNP) of the related miRNAs (let-7f rs10877887, let-7a-1 rs13293512, miR-133a-1 rs8089787, miR-133a-2 rs13040413, and miR-27a rs895819) and susceptibility to type 2 diabetes mellitus (T2DM), and its possible mechanisms.

Methods: Five SNPs in miRNAs (let-7f rs10877887, let-7a-1 rs13293512, miR-133a-1 rs8089787, miR-133a-2 rs13040413, and miR-27a rs895819) involved in the insulin signaling pathways were selected and genotyped in a case-control study that enrolled 371 T2DM patients and 381 non-diabetic controls. The individual SNP association analyses, interaction analyses of SNP-SNP, SNP-environmental factors were performed. The effect the risk-associated polymorphism on regulating its mature miRNA expression was also evaluated.

Results: In overall analyses, miR-133a-2 rs13040413 and let-7a-1 rs13293512 were related to the susceptibility to T2DM. In stratified analyses, miR-133a-2 rs13040413, let7a-1 rs13293512 and miR-27a rs895819 showed associations with T2DM in the age $\geq 60$ years subgroup. Moreover, let-7a-1 rs13293512 and miR-27a rs895819 showed associations with T2DM in male subgroup. In SNP-environmental factors interaction analyses, there were interaction effects of miR-133a-2 rs13040413 with dyslipidemia, let7a-1 rs13293512 with smoking, and let-7a-1 rs13293512 with dyslipidemia on T2DM. In SNP-SNP interaction analyses, there were also interaction effects of miR-133a-1 rs8089787 with let-7a-1 rs13293512, and miR-133a-1 rs8089787 with let-7f rs10877887 on T2DM. Furthermore, for miR-133a-2 rs13040413, the variant $T$ allele showed a trend toward decreased miR-133a expression in comparison with the wild $\mathrm{C}$ allele. For let-7a-1 rs13293512, the variant $\mathrm{C}$ allele expressed a lower let-7a compared to the wild $T$ allele. 
Conclusion: MiRNAs polymorphisms involved in the insulin signaling pathways and the interaction effects of SNP-SNP, SNP-environmental factors were related to T2DM susceptibility in a Chinese population.

Keywords: microRNA, polymorphism, type 2 diabetes mellitus, insulin signaling pathway, Chinese population

\section{INTRODUCTION}

Type 2 diabetes mellitus (T2DM) posting one of the most common and serious chronic diseases, is characterized sustained hyperglycemia (1). Insulin resistance, involving a defect in insulin secretion, insulin action, or both, is central to the etiology of T2DM (2). The underlying molecular mechanism for insulin resistance is only partially understood. Nevertheless, increasing evidences have showed that various microRNAs (miRNA) involved in regulating the main protein cascades in the insulin signaling pathways that affect insulin resistance, and therefore, the pathogenesis of T2DM, such as let-7f with insulin growth factor-1 receptor (IGF1R), let-7a with phosphatidylinositol 3-kinase (PI3K)/protein kinase B (AKT), miR-133a with glucose transporter 4 (GLUT4), and miR-27a with mammalian target of rapamycin (mTOR) (3-6).

It has been showed that IGF1R was the target of let-7f. Let-7f mimics could suppress IGF1R expression, and that let- $7 \mathrm{f}$ inhibitors could increase the expression level of IGF1R (3). Blood level of let-7f was down-regulated in T2DM subjects compared to controls (7). Moreover, let-7a is associated with PI3K/AKT signaling. Let-7a overexpression could decrease the expression levels of PI3K and p-AKT (4). Down-regulation of let-7a level was observed in T2DM subjects (8). Overexpression of miR-133a could decrease GLUT4 expression and reduced insulin-mediated glucose uptake (5). The circulatory miR-133a level was significantly higher in T2DM subjects than in controls. Further, there was a positive and significant correlation between miR-133a with fasting blood glucose (FBG) and glycated hemoglobin in the T2DM subjects (9). Elevated miR-27a could up-regulate mTOR phosphorylation level and enhance mTOR signaling (6). Increased blood level of miR-27a was identified in $\mathrm{T} 2 \mathrm{DM}$, and associated with measures of pancreatic $\beta$ cell function (10). Collectively, let-7f, let-7a, miR-133a, and miR27a have strong relationship with T2DM, both in underlying mechanisms and expression levels.

Single nucleotide polymorphisms (SNP) are variations in single nucleotides of genomic DNA sequence, which have modified potentials on gene functions (11). SNPs affect the miRNA binding efficiency, giving rise to increased or decreased miRNA regulation (12). We hypothesize that the SNPs in miRNA may impact the susceptibility to T2DM by miRNA dysregulation of mRNA degradation, translation, and expression. It has been showed that let-7f rs10877887 and let-7a-1 rs13293512 have relationships with stroke, depression, aneurysms and cancers (13-16). Especially, let7a-1 rs13293512 have binding sites with interleukin-6 (IL-6), a contributor of T2DM. The variant genotypes of let-7a-1 rs13293512 were associated with increased IL-6 expression (17). MiR-133a-1 rs8089787 are associated with asthma (18). MiR-133a-2 rs13040413 variant could increase levels of miR-133a (19). However, there was no study concerning about the associations between let- $7 \mathrm{f}$ rs10877887, let-7a-1 rs13293512, miR-133a-1 rs8089787 and miR133a-2 rs13040413 and T2DM. Furthermore, the relationships of miR-27a rs895819 and T2DM were controversial and inconclusive, which need further investigation (20). In addition, only a part of T2DM heritability can be interpreted by a single miRNA polymorphism study. SNP-SNP and SNP-environmental factors interactions may account for another missing part of the heritability. But there are lacking studies on the interactions of miRNA SNPs, as well as with environmental factors in T2DM.

Thus, the aim of our study is comprehensively investigating the effects of variations in miRNA genes involving in insulin signaling pathways, let-7f rs10877887, let-7a-1 rs13293512, miR133a-1 rs8089787, miR-133a-2 rs13040413, and miR-27a rs895819, the interactions of SNP-SNP, SNP-environmental factors on T2DM in a Chinese population, as well as the effect of the risk-associated polymorphism on regulating its mature miRNA expression.

\section{MATERIALS AND METHODS}

\section{Study Subjects}

A total of 752 participants were enrolled in this study, including 371 untreated T2DM patients and 381 non-diabetic controls. T2DM participants were recruited from inpatient and outpatient services at First Affiliated Hospital of China Medical University, and control participants from local community between August 2016 and December 2018. T2DM met the 1999 WHO criteria for diabetes: a FBG $\geq 7.0 \mathrm{mmol} / \mathrm{l}$ or a $2 \mathrm{~h}$ blood glucose $(2 \mathrm{hBG}) \geq$ $11.1 \mathrm{mmol} / 1$ (21). The non-diabetic controls were included as follows: a fasting glucose level $<6.1 \mathrm{mmol} / \mathrm{l}$ or $2 \mathrm{~h}$ glucose level $<$ $7.8 \mathrm{mmol} / \mathrm{l}$, no past history of diabetes and no family history of T2DM. Exclusion criteria were participants with type 1 diabetes or other special types of diabetes, malignant disease, liver and kidney disease, acute infections, or myocardial infarction. All participants provided written informed consent after receiving a detailed description of the study. The study was approved by the Institutional Ethics Committee of China Medical University. This study was carried out in accordance with the standard biosecurity and institutional safety procedures of China Medical University.

\section{Data Collection, Anthropometric Measure, and Laboratory Testing}

Data of sex, age, body mass index (BMI), smoking status, and alcohol consumption were collected from questionnaires. Smokers were defined as having smoked at least one cigarette 
per day for more than one year. Drinkers were defined as having consumed at least one alcoholic beverage a day for a minimum period of six months. Systolic blood pressure (SBP), diastolic blood pressure (DBP), blood glucose, total cholesterol (TC), high-density lipoprotein cholesterol (HDL), low-density lipoprotein cholesterol (LDL), and triglyceride (TG) were measured using standard laboratory procedures. Hypertension was defined as $\geq 140 / 90 \mathrm{mmHg}$ or any antihypertensive treatment. Dyslipidemia was defined as TC $\geq 5.17 \mathrm{mmol} / \mathrm{L}$, or $\mathrm{TG} \geq 1.70 \mathrm{mmol} / \mathrm{L}$, or $\mathrm{LDL} \geq 2.58 \mathrm{mmol} / \mathrm{L}$, or $\mathrm{HDL} \leq 0.91$ $\mathrm{mmol} / \mathrm{L}$ or under taking hypolipidemic drugs.

\section{SNP Selection and Genotyping}

Five tag-SNPs were identified as the following steps (22). First, the candidate SNPs were screened in the $5^{\prime}$ and $3^{\prime}$ region, mature sequence, pri-miRNA sequence, pre-miRNA sequence of the miRNA genes. Second, the candidate SNPs were selected in the combinations provided by the HapMap database (http://www. HapMap.org) and Haploview software 4.0 (http://www. broadinstitute.org/mpg/haploview). Third, the potential functions of the candidate SNPs were predict by the web-based analysis tools (SNPinfo, http://snpinfo.niehs.nih.gov; PolymiRTS Database 3.0, http://compbio.uthsc.edu; TFSEARCH 1.3, http:// www.cbrc.jp/research/db/TFSEARCH.html). Collectively, miR133a-1 rs8089787, miR-133a-2 rs13040413, let-7a-1 rs13293512, let-7f rs10877887, and miR-27a rs895819 were selected for genotyping.

Genomic DNA was extracted using the standard phenolchloroform method, and then diluted to a working concentration of 50ng/ $\mu \mathrm{l}$ before genotyping (23). The assays, primer design, and genotyping of candidate gene polymorphisms were performed by Baygene Biotechnology Company Limited (Shanghai, China) using the KASP method with SNPLine platform (LGC, United Kingdom). For genotyping quality controlling, 5\% samples were repeated genotyped and the results were $100 \%$ consistent.

\section{Transfection and Real-Time PCR Reaction for miRNA Expression}

The expression vectors pGCMV-rs13040413-C, pGCMVrs13040413-T (site-specific mutagenesis from $\mathrm{C}$ to $\mathrm{T}$ ), pGCMV-rs13293512-T, and pGCMV-rs13293512-C (sitespecific mutagenesis from $\mathrm{T}$ to $\mathrm{C}$ ) were synthesized by Genechem Company (Genechem Co. Ltd, Shanghai, China). The pGCMV-rs13040413-C, pGCMV-rs13040413-T, pGCMVrs13293512-T, pGCMV-rs13293512-C, and empty plasmids were transfected into human embryonic kidney (HEK) 293T cells, respectively. After $48 \mathrm{~h}$, the total RNA was extracted using TRIzol reagent (Invitrogen, USA). Real-time PCR was used to detect miR-133a and let-7a expression using TaqMan microRNA assay kits (ABI, USA). All experiments were carried out in triplicate.

\section{Statistical Analysis}

All statistical analyses were carried out with SPSS 16.0 software (SPSS, Chicago, IL, USA). Continuous variables were presented as mean $\pm \mathrm{SD}$ and compared by Student's $t$ test, and discrete variables, including the Hardy-Weinberg equilibrium (HWE) in control group, as frequencies and percentages and by Chi-square $\chi 2$ test. The association of SNPs and T2DM risk was assessed by the odds ratios (OR) with 95\% confidence intervals (CI) using multiple logistic regression analysis after adjustment for potential risk factors (age, gender, smoking, drinking, hypertension, and dyslipidemia). The interaction effects (SNP-SNP, SNPenvironment) were evaluated by the likelihood-ratio with a fully parameterized model. Differential expression of the wild type and mutant alleles was analyzed by Student's t test. A two-side $P$-value less than 0.05 was considered statistically significant.

\section{RESULTS}

\section{Demographic and Clinical Characteristics}

Demographic and clinical data are presented in Table 1. There were no significant differences in sex, age, BMI, smoking, and drinking between T2DM patients and controls $(P>0.05)$. T2DM patients had significant higher FBG, 2hBG, SBP, DBP, and TG and lower HDL when compared with controls $(P<0.05)$. The dyslipidemia rate was higher in T2DM patients compared to controls $(P<0.05)$.

\section{Association of MiRNA Polymorphisms With T2DM}

All genotypes were distributed in accordance with HardyWeinberg equilibrium $(P>0.05)$. As shown in Table 2 , miR133a-2 rs13040413 variant genotypes were significantly associated with an increased risk of T2DM (TT: OR $=2.18$, $P=0.028$; CT+TT: $\mathrm{OR}=1.38, P=0.040)$. let-7a-1 rs 13293512 variant genotypes were also associated with an increased risk of T2MD (CC: $\mathrm{OR}=1.61, P=0.026 ; \mathrm{TC}+\mathrm{CC}$ : $\mathrm{OR}=1.41$, $P=0.029)$. There was no overall genetic effect on T2DM for miR-133a-1 rs8089787, let-7f rs10877887, and miR-27a rs895819 $(P>0.05)$.

Moreover, stratified analyses were conducted to evaluate the associations between SNPs and T2DM by age and sex (Table 3). miR-133a-2 rs13040413 variant genotype was associated with an increased risk of T2MD in the age $\geq 60$ years subgroup (TT: $\mathrm{OR}=5.42, P=0.009)$. let-7a-1 rs13293512 variant genotypes were associated with an increased risk of T2MD in both the age $\geq 60$ years $(\mathrm{TC}: \mathrm{OR}=2.19, P=0.010$; CC: $\mathrm{OR}=2.40, P=0.005$; TC+CC: $\mathrm{OR}=3.20, P=0.002)$ and male subgroup (CC: $\mathrm{OR}=$ $1.87, P=0.040)$. miR-27a rs895819 variant genotypes were also associated with an increased risk of T2MD in both the age $\geq 60$ years (GG: $\mathrm{OR}=4.92, P=0.049$ ) and male subgroup (AG: $\mathrm{OR}=$ 1.53, $P=0.049$; TC+CC: $\mathrm{OR}=1.59, P=0.039)$. No statistical significant differences were observed between miR-133a-1 rs8089787 or let-7f rs10877887 polymorphisms and T2DM $(P>0.05)$.

\section{Interactions of MiRNA Polymorphisms and Environmental Factors in T2DM}

Interaction effects of miRNA polymorphisms and environmental factors in T2DM were also explored. The models included SNPs 
TABLE 1 | Demographics and clinical characteristics of type 2 diabetes mellitus cases and non-diabetic controls.

\begin{tabular}{|c|c|c|c|c|}
\hline Variables & T2DM (N=371) & controls $(\mathrm{N}=381)$ & $\mathrm{T} / \chi^{2}$ & $P$ value \\
\hline Sex, M/F (\%) & 196/175 (52.8/47.2) & 192/189 (50.4/49.6) & 0.447 & 0.504 \\
\hline Age (years) & $54.15 \pm 10.55$ & $54.71 \pm 8.96$ & -0.778 & 0.437 \\
\hline Body mass index (kg/m2) & $25.35 \pm 3.74$ & $25.38 \pm 3.28$ & -0.070 & 0.944 \\
\hline Smoking, Y/N (\%) & $113 / 258$ (30.4/69.6) & 98/283 (25.5/74.5) & 3.463 & 0.073 \\
\hline Drinking, Y/N (\%) & 75/296 (20.0/80.0) & 68/313 (17.6/82.4) & 0.689 & 0.407 \\
\hline Fasting blood glucose (mmol/L) & $8.48 \pm 2.77$ & $5.34 \pm 0.55$ & 21.591 & $<0.001$ \\
\hline 2h blood glucose (mmol/L) & $15.34 \pm 5.29$ & $6.29 \pm 1.92$ & 29.007 & $<0.001$ \\
\hline Systolic blood pressure (mmHg) & $138.301 \pm 21.07$ & $133.85 \pm 19.96$ & -2.969 & 0.003 \\
\hline Diastolic blood pressure (mmHg) & $87.49 \pm 13.07$ & $82.82 \pm 13.27$ & -4.865 & $<0.001$ \\
\hline Total cholesterol (mmol/L) & $5.08 \pm 1.15$ & $5.03 \pm 2.76$ & -0.332 & 0.740 \\
\hline Triglyceride (mmol/L) & $2.33 \pm 2.36$ & $1.44 \pm 1.03$ & 6.659 & $<0.001$ \\
\hline Low-density lipoprotein cholesterol (mmol/L) & $3.20 \pm 0.90$ & $3.17 \pm 0.96$ & -0.530 & 0.596 \\
\hline High-density lipoprotein cholesterol (mmol/L) & $1.24 \pm 0.54$ & $1.40 \pm 0.67$ & -3.452 & 0.001 \\
\hline Hypertension, Y/N (\%) & $157 / 214(42.3 / 57.7)$ & 140/241 (36.7/63.3) & 2.466 & 0.116 \\
\hline Dyslipidemia, Y/N (\%) & 235/136 (63.5/36.5) & 201/180 (52.8/47.2) & 8.921 & 0.003 \\
\hline
\end{tabular}

T2DM, type 2 diabetes mellitus.

TABLE 2 | Association of miRNA polymorphisms with type 2 diabetes mellitus.

\begin{tabular}{|c|c|c|c|c|c|c|}
\hline \multirow[t]{2}{*}{ Genotype } & & & \multicolumn{2}{|c|}{ Crude models } & \multicolumn{2}{|c|}{ Adjusted models } \\
\hline & & & OR(95\%Cl) & $P$ value & OR $(95 \% \mathrm{Cl})^{\mathrm{a}}$ & $P$ value \\
\hline \multicolumn{7}{|c|}{ miR-133a-1 rs8089787 } \\
\hline CT & vs & $\mathrm{CC}$ & $0.90(0.63,1.27)$ & 0.540 & $0.91(0.64,1.29)$ & 0.583 \\
\hline$\pi$ & vs & $\mathrm{CC}$ & $1.00(0.32,3.15)$ & 0.995 & 1.05(0.33, 3.32) & 0.929 \\
\hline $\mathrm{CT}+\mathrm{TT}$ & vs & $\mathrm{CC}$ & $0.90(0.64,1.27)$ & 0.558 & $0.91(0.65,1.29)$ & 0.603 \\
\hline \multicolumn{7}{|c|}{ miR-133a-2 rs13040413 } \\
\hline CT & vs & $\mathrm{CC}$ & $1.26(0.92,1.75)$ & 0.152 & $1.27(0.92,1.76)$ & 0.145 \\
\hline$\pi$ & vs & $\mathrm{CC}$ & $2.19(1.10,4.38)$ & 0.026 & 2.18(1.09, 4.36) & 0.028 \\
\hline $\mathrm{CT}+\mathrm{TT}$ & vs & $\mathrm{CC}$ & $1.38(1.01,1.87)$ & 0.041 & $1.38(1.02,1.87)$ & 0.040 \\
\hline \multicolumn{7}{|c|}{ let-7a-1 rs13293512 } \\
\hline TC & vs & $\Pi$ & $1.35(0.97,1.88)$ & 0.074 & $1.34(0.97,1.88)$ & 0.080 \\
\hline $\mathrm{CC}$ & vs & $\Pi$ & $1.61(1.06,2.44)$ & 0.026 & $1.61(1.06,2.44)$ & 0.026 \\
\hline $\mathrm{TC}+\mathrm{CC}$ & vs & $\Pi$ & $1.42(1.04,1.93)$ & 0.028 & $1.41(1.04,1.93)$ & 0.029 \\
\hline \multicolumn{7}{|c|}{ let-7f rs10877887 } \\
\hline TC & vs & $\pi$ & $1.22(0.90,1.66)$ & 0.197 & $1.21(0.89,1.65)$ & 0.219 \\
\hline $\mathrm{CC}$ & vs & $\pi$ & $0.83(0.52,1.31)$ & 0.414 & $0.82(0.52,1.30)$ & 0.404 \\
\hline $\mathrm{TC}+\mathrm{CC}$ & vs & $\pi$ & $1.12(0.84,1.49)$ & 0.442 & $1.11(0.83,1.48)$ & 0.467 \\
\hline \multicolumn{7}{|c|}{ miR-27a rs895819 } \\
\hline$A G$ & vs & $\mathrm{AA}$ & $1.26(0.93,1.71)$ & 0.138 & $1.26(0.93,1.71)$ & 0.134 \\
\hline GG & vS & $\mathrm{AA}$ & $1.27(0.72,2.23)$ & 0.408 & $1.26(0.72,2.22)$ & 0.421 \\
\hline$A G+G G$ & vs & $\mathrm{AA}$ & $1.26(0.94,1.68)$ & 0.116 & $1.26(0.94,1.68)$ & 0.117 \\
\hline
\end{tabular}

${ }^{a}$ ORs and $95 \% \mathrm{Cl}$ and corresponding $P$ values were calculated by logistic regression analysis adjusted by sex, age, smoking, drinking, hypertension, and dyslipidemia; OR, odds ratio; $\mathrm{Cl}$, confidence interval.

with smoking, drinking, hypertension, and dyslipidemia. As presented in Table 4, miR-133a-2 rs13040413 variant genotype had a positive interaction with dyslipidemia on T2DM $(\mathrm{OR}=10.26$, $P=0.004)$. let-7a-1 rs13293512 variant genotypes also had positive interactions with smoking (TC: $\mathrm{OR}=2.04, P=0.049$; TC+CC: $\mathrm{OR}=$ $2.15, P=0.027$ ) and dyslipidemia (CC: $\mathrm{OR}=1.93, P=0.040$; TC+CC: $\mathrm{OR}=2.56, P=0.030)$ on T2DM. However, there were no significant differences among other gene polymorphisms and environmental factors interactions $(P>0.05)$.

\section{MiRNA SNP-SNP Interactions in T2DM}

We further investigated miRNA SNP-SNP interaction effects in T2DM. The models included dominant genotypes of miRNA polymorphisms. It was showed that miR-133a-1 rs8089787 variant genotype and let-7a-1 rs13293512 variant genotype had a positive interaction effect in T2DM $(\mathrm{OR}=3.79, P=0.037)$. Moreover, there was a positive interaction effect of miR-133a-1 rs8089787 variant genotype and let-7f rs10877887 variant genotype in T2DM $(\mathrm{OR}=3.61, \mathrm{P}=0.035)$ (Table 5). No significant differences were observed among other miRNA SNP-SNP interactions $(P>0.05)$.

\section{The Effect of rs 13040413 and rs13293512 on miR-133a and let-7a Expression}

After 48 h, let-7a expression had statistical significance in HEK293T cell lines (Figure 1B). The variant $\mathrm{C}$ allele expressed a lower let- $7 \mathrm{a}$ when compared to the wild $\mathrm{T}$ allele $(2.51 \pm 0.22$ vs $3.11 \pm 0.28, \mathrm{P}=0.047)$. While $\mathrm{miR}-133 \mathrm{a}$ expression did not reach 
TABLE 3 | Stratified analysis of association between miRNA polymorphisms and type 2 diabetes mellitus.

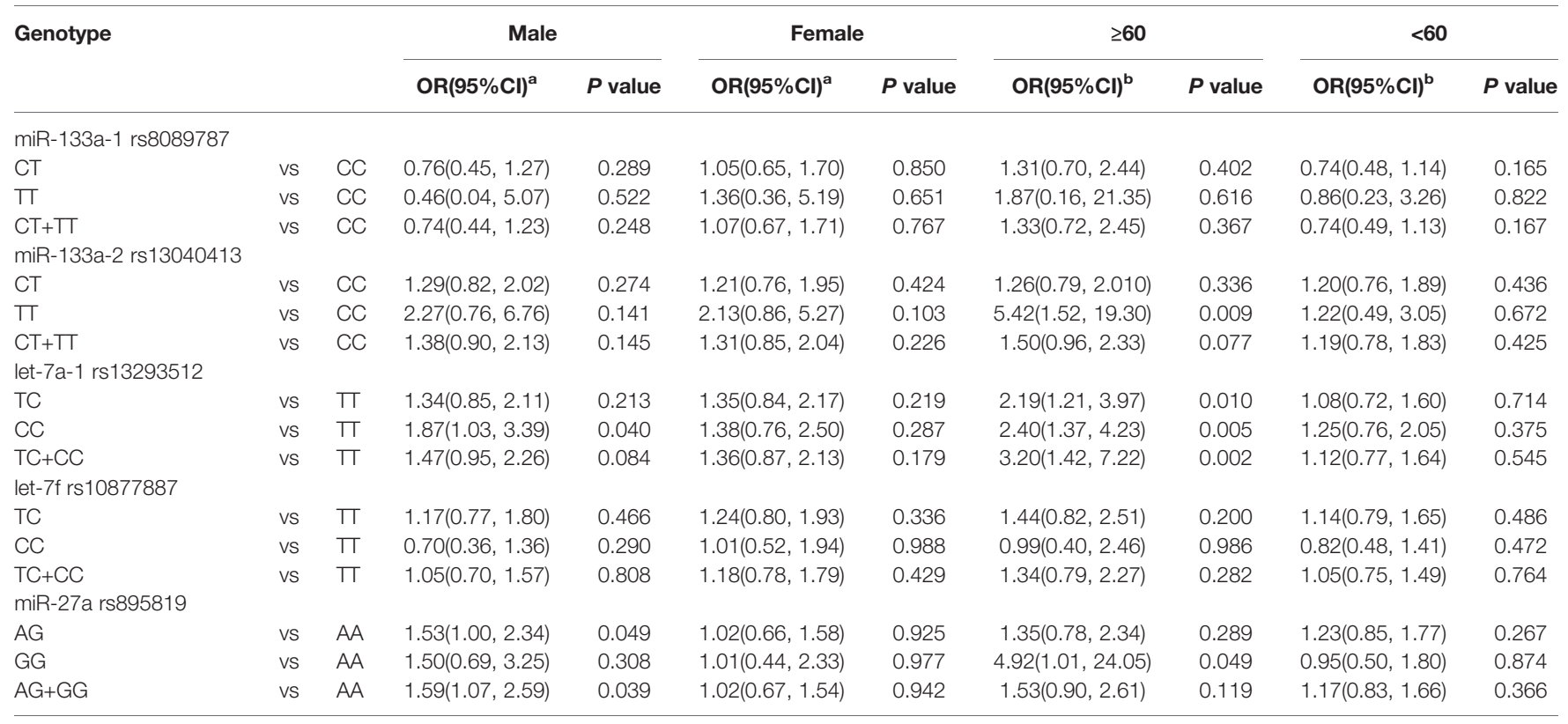

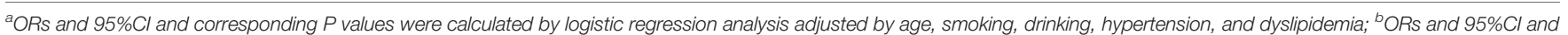
corresponding $P$ values were calculated by logistic regression analysis adjusted by sex, smoking, drinking, hypertension, and dyslipidemia; OR, odds ratio; Cl, confidence interval.

TABLE 4 | SNP-environmental factors interaction effects on type 2 diabetes mellitus.

\begin{tabular}{|c|c|c|c|c|c|c|c|c|c|c|}
\hline & & & \multicolumn{2}{|c|}{ Smoking (+) vs (-) } & \multicolumn{2}{|c|}{ Drinking (+) vs (-) } & \multicolumn{2}{|c|}{ Hypertension (+) vs (-) } & \multicolumn{2}{|c|}{ Dyslipidemia (+) vs (-) } \\
\hline & & & OR $(95 \% \mathrm{Cl})^{\mathrm{a}}$ & $P$ value & OR $(95 \% \mathrm{Cl})^{\mathrm{b}}$ & $P$ value & OR $(95 \% \mathrm{Cl})^{c}$ & $P$ value & OR $(95 \% \mathrm{Cl})^{d}$ & $P$ value \\
\hline \multicolumn{11}{|c|}{ miR-133a-1 rs8089787 } \\
\hline CT & vs & $\mathrm{CC}$ & $1.26(0.63,2.56)$ & 0.514 & $0.78(0.36,1.68)$ & 0.524 & $1.08(0.47,2.49)$ & 0.857 & $0.73(0.26,2.04)$ & 0.550 \\
\hline$\Pi \pi$ & vs & $\mathrm{CC}$ & $4.77(0.32,70.74)$ & 0.257 & $1.05(0.33,3.30)$ & 0.935 & $1.87(0.12,29.59)$ & 0.656 & $1.05(0.33,3.30)$ & 0.935 \\
\hline \multicolumn{10}{|c|}{ miR-133a-2 rs13040413 } & 0.538 \\
\hline CT & VS & $\mathrm{CC}$ & $1.02(0.54,1.96)$ & 0.946 & $1.42(0.70,2.90)$ & 0.333 & $1.82(0.86,3.88)$ & 0.120 & $0.87(0.38,1.96)$ & 0.730 \\
\hline$\Pi$ & vs & $\mathrm{CC}$ & $2.24(0.38,13.14)$ & 0.371 & $1.87(0.95,3.67)$ & 0.068 & $2.24(0.38,13.14)$ & 0.371 & $1.08(0.21,5.46)$ & 0.929 \\
\hline \multicolumn{11}{|c|}{ let-7a-1 rs13293512 } \\
\hline TC & vs & $\pi$ & $2.04(1.00,4.17)$ & 0.049 & $1.75(0.91,3.39)$ & 0.096 & $1.06(0.51,2.20)$ & 0.882 & $0.74(0.32,1.75)$ & 0.498 \\
\hline $\mathrm{CC}$ & vs & $\pi$ & $2.58(1.00,6.68)$ & 0.051 & $1.93(1.03,3.60)$ & 0.040 & $0.67(0.27,1.66)$ & 0.382 & $1.00(0.33,3.04)$ & 0.997 \\
\hline $\begin{array}{l}\mathrm{TC}+\mathrm{CC} \\
\text { let-7f rs10 }\end{array}$ & vs & $\pi$ & $2.15(1.09,4.23)$ & 0.027 & $2.56(1.09,6.01)$ & 0.030 & $0.93(0.47,1.84)$ & 0.828 & $0.80(0.35,1.80)$ & 0.583 \\
\hline $\mathrm{TC}$ & vS & $\pi$ & $1.40(0.76,2.58)$ & 0.286 & $1.50(0.76,2.97)$ & 0.240 & $0.81(0.41,1.61)$ & 0.551 & $0.55(0.25,1.21)$ & 0.136 \\
\hline $\mathrm{CC}$ & vs & $\pi$ & $1.74(0.69,4.42)$ & 0.241 & $2.33(0.81,6.69)$ & 0.116 & $1.11(0.38,3.21)$ & 0.850 & $1.46(0.46,4.66)$ & 0.520 \\
\hline $\begin{array}{l}\mathrm{TC}+\mathrm{CC} \\
\mathrm{miR}-27 \mathrm{a}\end{array}$ & vs & $\pi$ & $1.46(0.82,2.59)$ & 0.202 & $1.64(0.86,3.11)$ & 0.134 & $0.88(0.46,1.67)$ & 0.689 & $0.69(0.33,1.43)$ & 0.318 \\
\hline$A G$ & vs & $\mathrm{AA}$ & $1.11(0.61,2.05)$ & 0.730 & $0.97(0.49,1.91)$ & 0.926 & $1.18(0.59,2.34)$ & 0.640 & $1.30(0.59,2.84)$ & 0.518 \\
\hline$G G$ & vs & $\mathrm{AA}$ & $2.38(0.74,7.67)$ & 0.147 & $0.77(0.21,2.81)$ & 0.695 & $2.86(0.79,10.43)$ & 0.111 & $0.79(0.20,3.12)$ & 0.738 \\
\hline$A G+G G$ & vs & $\mathrm{AA}$ & $1.26(0.70,2.24)$ & 0.442 & $0.93(0.49,1.78)$ & 0.833 & $1.37(0.72,2.63)$ & 0.340 & $1.18(0.57,2.48)$ & 0.656 \\
\hline
\end{tabular}

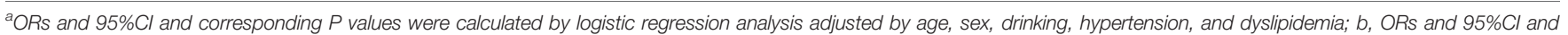

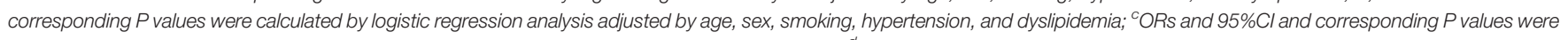

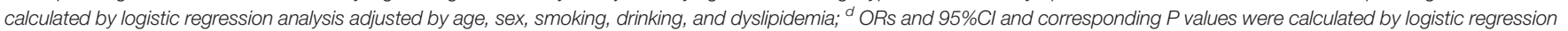
analysis adjusted by age, sex, smoking, drinking, and hypertension; SNP, single nucleotide polymorphism; OR, odds ratio; Cl, confidence interval.

statistical significance in HEK293T cell lines (Figure 1A). The variant $\mathrm{T}$ allele showed a trend toward decreased miR-133a expression in comparison with the wild $C$ allele $(0.76 \pm 0.10$ vs $0.99 \pm 0.12, \mathrm{P}=0.065$ ).

\section{DISCUSSION}

Alterations in the miRNA involved in the insulin signaling pathways play important roles in the pathogenesis of T2DM. 
TABLE 5 | SNP-SNP interaction effects on type 2 diabetes mellitus.

\begin{tabular}{|c|c|c|c|c|}
\hline \multicolumn{3}{|l|}{ Genetic model } & \multirow{2}{*}{$\begin{array}{c}\text { OR(95\% Cl) } \\
0.78(0.38,1.62)\end{array}$} & \multirow{2}{*}{$\frac{P \text { value }}{0.510}$} \\
\hline miR-133a-2 rs13040413 & $x$ & miR-133a-1 rs8089787 & & \\
\hline $\mathrm{CT}+\mathrm{TT}$ vs CC & & $\mathrm{CT}+\mathrm{TT}$ vs CC & & \\
\hline miR-133a-2 rs13040413 & $x$ & let-7a-1 rs13293512 & $1.00(0.51,1.95)$ & 0.997 \\
\hline $\mathrm{CT}+\mathrm{TT}$ vs CC & & $\mathrm{TC}+\mathrm{CC}$ vs TT & & \\
\hline miR-133a-2 rs13040413 & $x$ & let-7f rs10877887 & $1.42(0.77,2.63)$ & 0.265 \\
\hline $\mathrm{CT}+\mathrm{TT}$ vs CC & & $\mathrm{TC}+\mathrm{CC}$ vs TT & & \\
\hline miR-133a-2 rs13040413 & $x$ & miR-27a rs895819 & $0.84(0.45,1.56)$ & 0.581 \\
\hline $\mathrm{CT}+\mathrm{TT}$ vs CC & & $A G+G G$ vs $A A$ & & \\
\hline miR-133a-1 rs8089787 & $\times$ & let-7a-1 rs13293512 & $3.79(1.60,8.41)$ & 0.037 \\
\hline $\mathrm{CT}+\mathrm{TT}$ vs CC & & $\mathrm{TC}+\mathrm{CC}$ vs TT & & \\
\hline miR-133a-1 rs8089787 & $x$ & let-7f rs10877887 & $3.61(2.01,5.38)$ & 0.035 \\
\hline $\mathrm{CT}+\mathrm{TT}$ vs CC & & $\mathrm{TC}+\mathrm{CC}$ vs TT & & \\
\hline miR-133a-1 rs8089787 & $\times$ & miR-27a rs895819 & $1.07(0.54,2.13)$ & 0.844 \\
\hline $\mathrm{CT}+\mathrm{TT}$ vs CC & & $A G+G G$ vs $A A$ & & \\
\hline let-7a-1 rs13293512 & $x$ & let-7f rs10877887 & $0.87(0.46,1.62)$ & 0.654 \\
\hline $\mathrm{TC}+\mathrm{CC}$ vs TT & & $\mathrm{TC}+\mathrm{CC}$ vs TT & & \\
\hline let-7a-1 rs13293512 & $\times$ & miR-27a rs895819 & $0.78(0.42,1.47)$ & 0.443 \\
\hline $\mathrm{TC}+\mathrm{CC}$ vs TT & & $A G+G G$ vs $A A$ & & \\
\hline let-7f rs10877887 & $x$ & miR-27a rs895819 & $1.25(0.70,2.22)$ & 0.458 \\
\hline $\mathrm{TC}+\mathrm{CC}$ vs TT & & $A G+G G$ vs $A A$ & & \\
\hline
\end{tabular}

${ }^{a} \mathrm{ORs}$ and $95 \% \mathrm{Cl}$ and corresponding $P$ values were calculated by logistic regression analysis adjusted by age, sex, smoking, drinking, hypertension, and dyslipidemia; SNP, single nucleotide polymorphism; OR, odds ratio; $\mathrm{Cl}$, confidence interval.
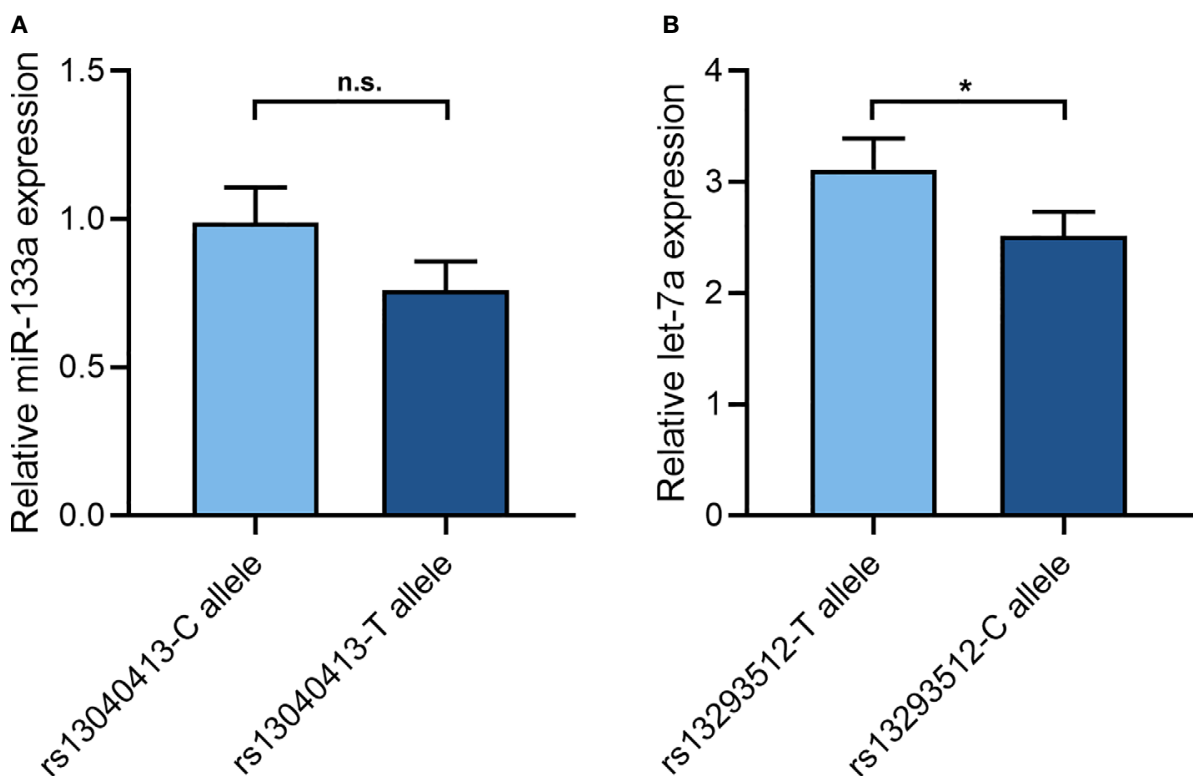

FIGURE 1 | The effect of rs13040413 and rs13293512 on miR-133a and let-7a expression. (A) The cell miR-133a expression by different miR-133a-2 rs13040413 plasmids. (B) The cell let-7a expression by different let-7a-1 rs13293512 plasmids. ${ }^{\star} P<0.05$. n.s., no significance.

SNPs in these miRNA may down-regulate or up-regulate miRNA expression in T2DM (24). The present study focused on the relation of SNPs of miRNA involved in the insulin signaling pathways, miR-133a-1 rs8089787, miR-133a-2 rs13040413, let-7a-1 rs13293512, let-7f rs10877887, and miR27a rs895819, with T2DM, and the interaction effects of SNPSNP and SNP-environmental factors on T2DM, as well as the effect of the risk-associated polymorphism on regulating its mature miRNA expression. To our knowledge, this is the first to comprehensively report the issues.

Our findings revealed that miR-133a-2 rs13040413 and let-7a-1 rs13293512 were associated with increased T2DM risk. For miR-133a-2 rs13040413, the variant $\mathrm{T}$ allele showed a trend toward decreased miR-133a expression in comparison with the 
wild C allele. For let-7a-1 rs13293512, the variant C allele expressed a lower let-7a compared to the wild $\mathrm{T}$ allele. It has been suggested that miR-133a can affect IGF1R and GLUT4 expression in the insulin signaling pathways. Gong et al. verified that IGF1R gene is a direct target of miR-133a using luciferase reporter assays (25). MiR133a can directly suppress the expression of IGF1R through translational repression. Moreover, miR-133a has been reported to act indirectly upon GLUT4 expression. MiR-133a can target the Krueppel-like factor 15 (Klf15) mRNA, inhibiting this transcriptional factor, which is an enhancer of GLUT4 expression, thus leading to the reduction in GLUT4 expression and in insulin stimulated glucose uptake (5). MiR-133a not only showed differential expression in blood level of T2DM, but also in tissue levels of T2DM complications, such as kidney, heart, sciatic nerve, and skeletal muscle (26-29). MiR-133a-2 rs13040413 variant could also increase levels of miR-133a (19). All the above contribute to the underlying mechanisms of the association between miR-133a-2 rs13040413 and T2DM. With regard to let-7a-1 rs13293512, it has been showed that the targets of let-7a is IGF1R, insulin receptor substrate 2 (IRS2) and PI3K/AKT of insulin signaling pathways. Exogenous expression of let-7a can suppress the expression of IGF1R (30). Meanwhile, inhibition of let-7a is sufficient to enhance the expression of IRS2 (31). Wang et al. has also showed that transfection of let-7a mimics can lead to the inhibition of the PI3K/ $\mathrm{AKT}$, and transfection of let-7a inhibitors may activate the PI3K/ AKT through the increase in PI3K and AKT levels (4). IGF1R, IRS2, and PI3K/AKT are known to mediate both insulin signaling pathways and insulin-induced cell dysregulations. As a functional polymorphism, let-7a-1 rs13293512 can substantially reduce the transcription activity of let-7, as well as directly upregulate IL-6 expression (17). The deep understanding of the potential mechanisms will yield further insights into the relationship of let7a-1 rs13293512 and T2DM.

Additionally, in the stratified analyses, miR-133a-2 rs13040413, let-7a-1 rs13293512, and miR-27a rs895819 were associated with increased T2DM risk in older subjects (age $\geq 60$ years), suggesting that age effect was dominant cause of T2DM in older subjects. Accumulated exposure to insulin disturbance caused by these three SNPs and weak immune system were expected in older subjects with T2DM. Let-7a-1 rs13293512 and miR-27a rs895819 were associated with increased T2DM risk in male subjects. Hormonal differences between males and females may elucidate sex-specific variation in T2DM. There is evidence to show bidirectional signaling cross-talk between let-7a, miR$27 \mathrm{a}$, and estrogen receptors $(32,33)$. A role for estrogen in the regulation of glucose metabolism provides a potential biologic explanation to our results (34).

Being a multifactorial disease, there might be complex interactions between the risk allele and confounding factors in T2DM. In our study, interaction effects on T2DM have been demonstrated between miR-133a-2 rs13040413 or let-7a-1 rs13293512 and dyslipidemia. It has been showed that the expression levels of miR-133a and let-7a were in a dysregulation pattern under high lipid condition (35). Evidence also showed that miR-133a and let-7a were also related to lipid accumulation (36, 37). Additionally, we observed there was interaction effect between let-7a-1 rs13293512 and smoking on T2DM. Banerjee A. showed that let-7a was differentially expressed between the smoking and nonsmoking subjects (38). Let-7a showed a correlation with haemoglobin adduct biomarkers of tobacco exposure. Moreover, besides miR-133a and let-7a, dyslipidemia and smoking have been known as high risk factors of T2DM. Collectively, we assumed that miR-133a and let-7a had cross-talk with dyslipidemia or smoking, which could explain the phenomenon of interaction effects between miR-133a-2 rs13040413 or let-7a-1 rs13293512 and dyslipidemia or smoking on T2DM.

In our study, we also detected interaction effects of miR-133a-1 rs8089787 and let-7a-1 rs13293512 or let-7f rs10877887 on T2DM. This phenomenon is defined as epistatic effect, which usually accounts for missing or underestimated heritability when one single gene is included in disease susceptibility (39). Likely, miR133a rs 8089787 had no effect on T2DM. In contrast, miR-133a-1 rs8089787 and let-7a-1 rs13293512 or let-7f rs10877887 worked together to generate interaction effects on T2DM. We assumed that the functional effect of miR-133a, let-7a, and let-7f in the insulin signaling pathway might account for the observed interaction effect. As described above, miR-133a and let-7a were involved in the insulin signaling pathway and the pathogenesis of T2DM. As for let$7 \mathrm{f}$, it has been reported that let-7f could target multiple key genes related to insulin signaling pathway. Mellios et al. reported that let$7 \mathrm{f}$ could inhibit IGF1 expression, and inhibition of let-7f could significantly up-regulate levels of IGF1 mRNA (40). Hu et al. showed that let-7f mimics suppressed IGF1R expression, and let$7 f$ inhibitors increased the expression level of IGF1R (3). Furthermore, Wang et al. revealed that insulin-like growth factor 2 mRNA binding protein 1 (IGF2BP1) was potential target of let-7f. Let-7f could suppress IGF2BP1 expression (41). Combined, any genetic mutation in the insulin signaling pathway, like miR-133a-1 rs8089787, let-7a-1 rs13293512, and let-7f rs10877887, could potentially alter the action of each other so as to influence insulin function in pathogenesis of T2DM.

Several limitations of this study should be noted. First, the sample size in this study was relatively small, which might restrict the ability to explore weak genetic effect on T2DM. Prospective studies consisting of larger-scale sample and multicenter surveys are necessary to validate the findings of miRNA polymorphisms on T2DM. Second, only miRNA polymorphisms were included in this study. Further studies should involve SNPs in the miRNA binding site of potential targeted genes, which could deeply explored the SNP-SNP interactions in miRNA regulatory pathways. Third, considering the clinical significance of the study, the more comprehensive functional and molecular experiments of the mentioned miRNA polymorphisms on T2DM would be of great significance for clinical practice and may be an important future direction.

\section{CONCLUSIONS}

This study, for the first time, investigated the relationships of miRNA polymorphisms involving in insulin signaling pathways and T2DM, and reported that miR-133a-2 rs13040413, let-7a-1 rs13293512, and miR-27a rs895819 were related to the 
susceptibility to T2DM in overall or stratified analyses in a Chinese population. For miR-133a-2 rs13040413, the variant $\mathrm{T}$ allele showed a trend toward decreased miR-133a expression in comparison with the wild $\mathrm{C}$ allele. For let-7a-1 rs13293512, the variant $\mathrm{C}$ allele expressed a lower let-7a compared to the wild $\mathrm{T}$ allele. Novel interaction effects on T2DM were revealed among miR-133a-2 rs13040413 with dyslipidemia, let-7a-1 rs13293512 with smoking, let-7a-1 rs13293512 with dyslipidemia, miR133a-1 rs8089787 with let-7a-1 rs13293512, and miR-133a-1 rs 8089787 with let-7f rs10877887. Future large-scale studies and more comprehensive mechanism experiments are required.

\section{DATA AVAILABILITY STATEMENT}

The datasets presented in this study can be found in online repositories. The names of the repository/repositories and accession number(s) can be found in the article/supplementary material.

\section{ETHICS STATEMENT}

The studies involving human participants were reviewed and approved by the Institutional Review Board of China Medical University. The patients/participants provided their written informed consent to participate in this study.

\section{REFERENCES}

1. Liu MH, Li Y, Han L, Zhang YY, Wang D, Wang ZH, et al. Adipose-derived stem cells were impaired in restricting $\mathrm{CD} 4(+) \mathrm{T}$ cell proliferation and polarization in type 2 diabetic ApoE(-/-) mouse. Mol Immunol (2017) 87:152-60.

2. Chiasson JL, Rabasa-Lhoret R. Prevention of type 2 diabetes: insulin resistance and beta-cell function. Diabetes (2004) 53 Suppl 3:S34-8.

3. Hu W, Li T, Hu R, Wu L, Li M, Meng X. MicroRNA let-7a and let-7f as novel regulatory factors of the sika deer (Cervus nippon) IGF-1R gene. Growth Factors (2014) 32:27-33.

4. Wang T, Zhu H, Yang S, Fei X. Let7a5p may participate in the pathogenesis of diabetic nephropathy through targeting HMGA2. Mol Med Rep (2019) 19:4229-37.

5. Horie T, Ono K, Nishi H, Iwanaga $\mathrm{Y}$, Nagao K, Kinoshita M, et al. MicroRNA-133 regulates the expression of GLUT4 by targeting KLF15 and is involved in metabolic control in cardiac myocytes. Biochem Biophys Res Commun (2009) 389:315-20.

6. Zhang E, Chen Q, Wang J, Li D, Wan Z, Ju X. Protective role of microRNA27a upregulation and HSP90 silencing against cerebral ischemia-reperfusion injury in rats by activating $\mathrm{PI} 3 \mathrm{~K} / \mathrm{AKT} / \mathrm{mTOR}$ signaling pathway. Int Immunopharmacol (2020) 86:106635.

7. Santovito D, De Nardis V, Marcantonio P, Mandolini C, Paganelli C, Vitale E, et al. Plasma exosome microRNA profiling unravels a new potential modulator of adiponectin pathway in diabetes: effect of glycemic control. J Clin Endocrinol Metab (2014) 99:E1681-5.

8. Liang Z, Gao KP, Wang YX, Liu ZC, Tian L, Yang XZ, et al. RNA sequencing identified specific circulating miRNA biomarkers for early detection of diabetes retinopathy. Am J Physiol Endocrinol Metab (2018) 315:E374-85.

9. Ghasemi H, Karimi J, Khodadadi I, Tavilani H. Correlation between miR-103 and miR-133a Expression and the Circulating ANGPTL8 in Type 2 Diabetic Patients and Healthy Control Subjects. Clin Lab (2019) 65:19436.

10. Nunez Lopez YO, Garufi G, Seyhan AA. Altered levels of circulating cytokines and microRNAs in lean and obese individuals with prediabetes and type 2 diabetes. Mol Biosyst (2016) 13:106-21.

\section{AUTHOR CONTRIBUTIONS}

DS conceptualized and supervised the study. JY and CM designed the study together with DS, ZZ, YZ, and RB carried out genomic DNA extraction and SNP genotyping. RY and ZS participated in the clinical data and blood sample collections. DS and YZ did the statistical analyses. All authors contributed to data interpretation. DS, ZZ, and YZ wrote the paper with input from all authors. JY and CM revised the manuscript. All authors contributed to the article and approved the submitted version.

\section{FUNDING}

This work was supported by research grants from the National Natural Science Foundation of China (81701703 to DS), and the Shenyang Science and Technology Plan Project (19-112-4-061 to JY).

\section{ACKNOWLEDGMENTS}

The authors thank all the participants for their cooperation and are grateful for the support of Department of cardiovascular ultrasound, Department of Endocrinology and Metabolism, First Affiliated Hospital of China Medical University.

11. Shen H, Li J, Zhang J, Xu C, Jiang Y, Wu Z, et al. Comprehensive characterization of human genome variation by high coverage wholegenome sequencing of forty four Caucasians. PloS One (2013) 8:e59494.

12. Ghaedi H, Bastami M, Zare-Abdollahi D, Alipoor B, Movafagh A, Mirfakhraie R, et al. Bioinformatics prioritization of SNPs perturbing microRNA regulation of hematological malignancy-implicated genes. Genomics (2015) 106:360-6.

13. Zhang L, Yang J, Xue Q, Yang D, Lu Y, Guang X, et al. An rs13293512 polymorphism in the promoter of let-7 is associated with a reduced risk of ischemic stroke. J Thromb Thrombolysis (2016) 42:610-5.

14. Liang Y, Zhao G, Sun R, Mao Y, Li G, Chen X, et al. Genetic variants in the promoters of let-7 family are associated with an increased risk of major depressive disorder. J Affect Disord (2015) 183:295-9.

15. Sima X, Sun H, Zhou P, You C. A Potential Polymorphism in the Promoter of Let-7 is Associated With an Increased Risk of Intracranial Aneurysm: A CaseControl Study. Medicine (2015) 94:e2267.

16. Liu J, Ni S. Association between genetic polymorphisms in the promoters of let-7 and risk of cervical squamous cell carcinoma. Gene (2018) 642:256-60.

17. Dong H, Huang Z, Zhang H, Xiao Z, Liu Q. Rs13293512 polymorphism located in the promoter region of let-7 is associated with increased risk of radiation enteritis in colorectal cancer. J Cell Biochem (2018) 119:6535-44.

18. Zhou PP, Li Y, Ma ZD, Li ZY, Chen FY, Jiang YX. Single nucleotide polymorphisms in the promoter region of mir-133a-1 and in pre-mir-152 rs1707 may contribute to the risk of asthma in a Chinese Han population. Eur Rev Med Pharmacol Sci (2016) 20:2642-9.

19. Ohanian M, Humphreys DT, Anderson E, Preiss T, Fatkin D. A heterozygous variant in the human cardiac miR-133 gene, MIR133A2, alters miRNA duplex processing and strand abundance. BMC Genet (2013) 14:18.

20. Li G, Liu B, Jiang Q, Zhang J, Xin S, Xu K. The association of two common polymorphisms in miRNAs with diabetes mellitus: A meta-analysis. Medicine (2019) 98:e17414.

21. Alberti KG, Zimmet PZ. Definition, diagnosis and classification of diabetes mellitus and its complications. Part 1: diagnosis and classification of diabetes 
mellitus provisional report of a WHO consultation. Diabet Med (1998) 15:539-53.

22. Sun D, Sun L, Xu Q, Gong Y, Wang H, Yang J, et al. SNP-SNP Interaction between TLR4 and MyD88 in Susceptibility to Coronary Artery Disease in the Chinese Han Population. Int J Environ Res Public Health (2016) 13:278.

23. Xu Q, Yuan Y, Sun LP, Gong YH, Xu Y, Yu XW, et al. Risk of gastric cancer is associated with the MUC1 568 A/G polymorphism. Int J Oncol (2009) 35:1313-20.

24. Zhang Y, Bai R, Liu C, Ma C, Chen X, Yang J, et al. MicroRNA singlenucleotide polymorphisms and diabetes mellitus: A comprehensive review. Clin Genet (2019) 95:451-61.

25. Gong Y, Ren J, Liu K, Tang LM. Tumor suppressor role of miR-133a in gastric cancer by repressing IGF1R. World J Gastroenterol (2015) 21:2949-58.

26. Lee WC, Li LC, Ng HY, Lin PT, Chiou TT, Kuo WH, et al. Urinary Exosomal MicroRNA Signatures in Nephrotic, Biopsy-Proven Diabetic Nephropathy. J Clin Med (2020) 9.

27. Nandi SS, Zheng H, Sharma NM, Shahshahan HR, Patel KP, Mishra PK. Lack of miR-133a Decreases Contractility of Diabetic Hearts: A Role for Novel Cross Talk Between Tyrosine Aminotransferase and Tyrosine Hydroxylase. Diabetes (2016) 65:3075-90.

28. Chang LL, Wang HC, Tseng KY, Su MP, Wang JY, Chuang YT, et al. Upregulation of miR-133a-3p in the Sciatic Nerve Contributes to Neuropathic Pain Development. Mol Neurobiol (2020) 57:3931-42.

29. Frias Fde T, de Mendonca M, Martins AR, Gindro AF, Cogliati B, Curi R, et al. MyomiRs as Markers of Insulin Resistance and Decreased Myogenesis in Skeletal Muscle of Diet-Induced Obese Mice. Front Endocrinol (2016) 7:76.

30. Tian B, Huo N, Li M, Li Y, He Z. let-7a and its target, insulin-like growth factor 1 receptor, are differentially expressed in recurrent prostate cancer. Int $J$ Mol Med (2015) 36:1409-16.

31. Gurung B, Muhammad AB, Hua X. Menin is required for optimal processing of the microRNA let-7a. J Biol Chem (2014) 289:9902-8.

32. Sun X, Qin S, Fan C, Xu C, Du N, Ren H. Let-7: a regulator of the ERalpha signaling pathway in human breast tumors and breast cancer stem cells. Oncol Rep (2013) 29:2079-87.

33. Ali HO, Arroyo AB, Gonzalez-Conejero R, Stavik B, Iversen N, Sandset PM, et al. The role of microRNA-27a/b and microRNA-494 in estrogen-mediated downregulation of tissue factor pathway inhibitor alpha. J Thromb Haemost (2016) 14:1226-37.
34. Weigt C, Hertrampf T, Flenker U, Hulsemann F, Kurnaz P, Fritzemeier KH, et al. Effects of estradiol, estrogen receptor subtype-selective agonists and genistein on glucose metabolism in leptin resistant female Zucker diabetic fatty (ZDF) rats. J Steroid Biochem Mol Biol (2015) 154:12-22.

35. Youssef EM, Elfiky AM, Soliman B, Abu-Shahba N, Elhefnawi MM. Expression profiling and analysis of some miRNAs in subcutaneous white adipose tissue during development of obesity. Genes Nutr (2020) 15:8.

36. Peng XP, Huang L, Liu ZH. miRNA-133a attenuates lipid accumulation via TR4-CD36 pathway in macrophages. Biochimie (2016) 127:79-85.

37. Kim YS, Nam HJ, Han CY, Joo MS, Jang K, Jun DW, et al. LXRalpha activation inhibits autophagy and lipophagy in hepatocytes by dysregulating ATG4B and Rab-8B, reducing mitochondrial fuel oxidation. Hepatology (2020).

38. Banerjee A, Waters D, Camacho OM, Minet E. Quantification of plasma microRNAs in a group of healthy smokers, ex-smokers and non-smokers and correlation to biomarkers of tobacco exposure. Biomarkers (2015) 20:123-31.

39. Eichler EE, Flint J, Gibson G, Kong A, Leal SM, Moore JH, et al. Missing heritability and strategies for finding the underlying causes of complex disease. Nat Rev Genet (2010) 11:446-50.

40. Mellios N, Woodson J, Garcia RI, Crawford B, Sharma J, Sheridan SD, et al. beta2-Adrenergic receptor agonist ameliorates phenotypes and corrects microRNA-mediated IGF1 deficits in a mouse model of Rett syndrome. Proc Natl Acad Sci U S A (2014) 111:9947-52.

41. Wang Y, Chen X, Zhang Y, Song J. Potential proteins targeted by let-7f-5p in HeLa cells. Biosci Trends (2017) 11:363-5.

Conflict of Interest: The authors declare that the research was conducted in the absence of any commercial or financial relationships that could be construed as a potential conflict of interest.

Copyright (ङ 2021 Zhu, Zhang, Bai, Yang, Shan, Ma, Yang and Sun. This is an open-access article distributed under the terms of the Creative Commons Attribution License (CC BY). The use, distribution or reproduction in other forums is permitted, provided the original author(s) and the copyright owner(s) are credited and that the original publication in this journal is cited, in accordance with accepted academic practice. No use, distribution or reproduction is permitted which does not comply with these terms. 